\title{
Neurochemical Modulation Frequency Band of Hippocampal Theta Rhythm
}

\author{
Rafiga Mazahir Baghirova* \\ Azerbaijan State Academy of Physical Education and Sport, Azerbaijan Republic
}

*Corresponding author: Rafiga Mazahir Baghirova, Azerbaijan State Academy of Physical Education and Sport, Baku, Azerbaijan Republic

\section{ARTICLE INFO}

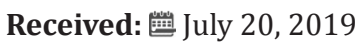

Published: 慧 July 30, 2019

Citation: Rafiga Mazahir Baghirova. Neurochemical Modulation Frequency Band of Hippocampal Theta Rhythm. Biomed J Sci \& Tech Res 20(2)-2019. BJSTR. MS.ID.003410.

Keywords: Hippocampal Theta Rhythm; Dorsal and Ventral Amigdalofugal Pathway; Electrical and chemical stimulation; Destruction

\begin{abstract}
In chronic experiments with rabbits have shown that electrical destruction of dorsal amygdalofugal pathways leads to complete and persisted blockade of hippocampal theta rhythm in contrast to ventral one. In intact animals, electro- and chemostimulation of various limbic structures of the brain (amygdala, hypothalamus, reticular formation, medial septum nucleus) lead to the formation of well pronounced theta rhythm in hippocampus, but after destruction of the dorsal amygdalofugal pathway any thetarhythm in this structure is not observed. Restoration of hippocampal EEG took place under intrahippocampal application of carbocholine and strychnine. It is proposed that one of the necessary conditions for the regulation of excitability of hippocampal neurons is the integrity of dorsal amygdalofugal pathways by means of which regulatory influence of amygdale on the hippotalamic neurosecretory cells is realized.
\end{abstract}

\section{Introduction}

The phenomenon of disappearance of the theta rhythm after lesioning of the septum and fornix has in its time stimulated a series of reports describing attempts to understand the roles of various structures in determining the electrical activity of the hippocampus. The medial septum nucleus, standing at the entrance to the hippocampus, demonstrates the importance of education [14]. In addition to the data indicative of the pacemaker role of the septum, there are works in which he showed a definite role of stemdiencephalon structures in mechanisms of formation hippokamal theta rhythm: of great importance given to the reticular formation [5], the hypothalamus [6], the thalamus [6], the locus cereleus [7], nucleus raphe [1], etc.

In recent experimental studies have shown that the medial septum nucleus receives phases already encoded information from the uplink system, the frequency of which determines and the frequency of discharges of the hippocampal theta rhythm. There is evidence that this information comes from the supramammilyar nucleus of the hypothalamus [8]. Our research has previously been shown that the destruction of Dorsal
Amigdalofugal Pathway (DAP), in contrast to the Ventral (VAP), resulting in complete and irreversible blockade of hippocampus theta rhythm [9]. To clarify the reasons for the profound changes we have conducted electrical and chemical stimulyation the limbic structures of the brain (the amygdale, hypothalamus, reticular formation, the medial nucleus of the septum, the hippocampus) before and after the destruction of the DAP.

\section{Methods}

Experiments were carried out on 16 mature rabbits weighing 2.5-3.0 kg. The recording of electrical activity from the hippocampus and the medial nucleus of the septum were performed 18-27 days after the destruction of the DAP. The EHipG was recorded from the dorsal hippocampus (the CA1field: P 3.0, L 2.0, H18.0, and the CA3 field: P 0 2.0, L6.0, H17.0) and from the medial nucleus of the septum (A-3.0; L0.5; H10.5) on a Medikor EEG-16E encephalograph with the use of needle electrodes insulated except the tip. Spectral EHipG analysis was performed using standard electroencephalographic approaches. Test substances were strychnine (1\%), carbachol (0.5-1.5 mcg), serotonin (5-50 mcg), and noradrenaline (15- 
$20 \mathrm{mcg}$ ) applied in volumes of $5-6 \mathrm{ml}$ via a chemotrode implanted in field CA3. Electrocoagulation of the dorsal amygdalofugal pathway (stria terminalis) was performed using electrodes implanted in the precommissural area (A-1, L5, H18) with currents of up to $1.0 \mathrm{~mA}$ for 15-25 sec. Electrical stimulation of extrahippocampal structures (reticular formation: P9, L2.5, H18.2; basolateral nucleus of the amygdala: A-1, L5, H18; central nucleus of the amygdala: A-1, L5, H16; supraoptic nucleus of the hypothalamus: A-3, L2.2, H15.8; ventromedial nucleus of the hypothalamus: AP0, L0.5, H17; medial mammilary nucleus of the hypothalamus: P2, L0.5, H18.5) and field CA3 was performed using an ÉSU-1 stimulator with square-wave impulses at frequencies of 5-100 per sec, amplitude 2-4 V, and duration $0.15 \mathrm{msec}$, for $15-30 \mathrm{sec}$; a histogram method was used for amplitude- frequency analysis of the EEG, as described by Fujimori et al. [3].

\section{Results and Discussion}

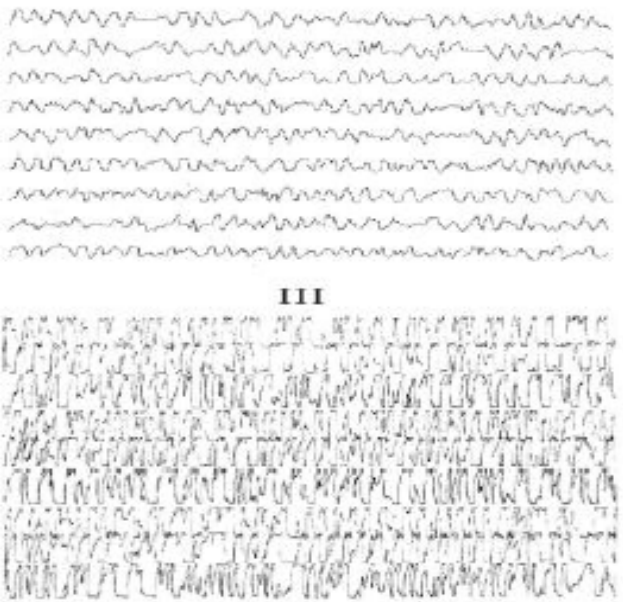

II

Whandmow

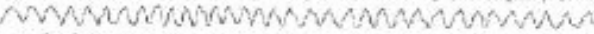

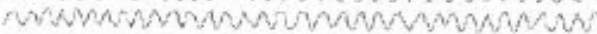

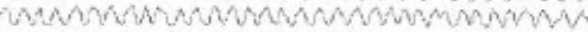

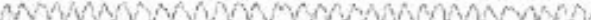

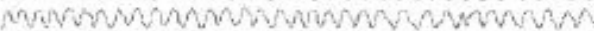

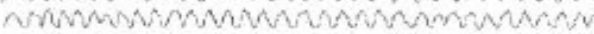

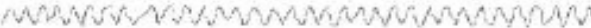

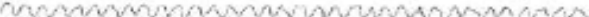

IV

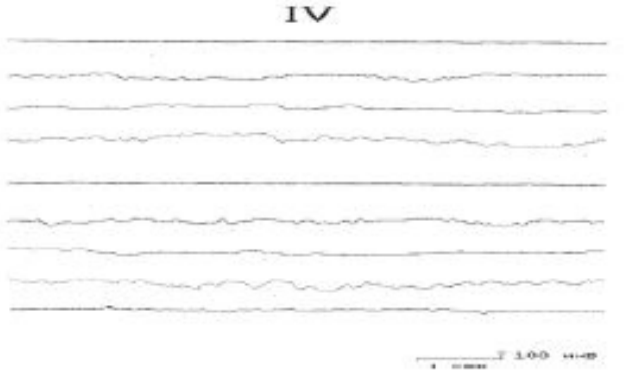

Figure 1: The effect of the introduction of serotonin into the nucleus of the hypothalamus on the electrical activity of the hippocampus before and after the destruction of the DAP. I - background activity; II - after application of 10-30 $\mu \mathrm{g}$ of serotonin; III- after application of 50-100 $\mu \mathrm{g}$ of serotonin; IV-application of serotonin on the background of the destruction of the DAP.1,2) Field CA1;3, 4) field CA3 of the ipsi-and contralateral hemispheres;5,6) ventral hippocampus of the ipsi- and contralateral hemispheres; 7,8)dentate gyrus of the ipsi- and contralateral hemispheres; 9) medial nucleus of the septum.

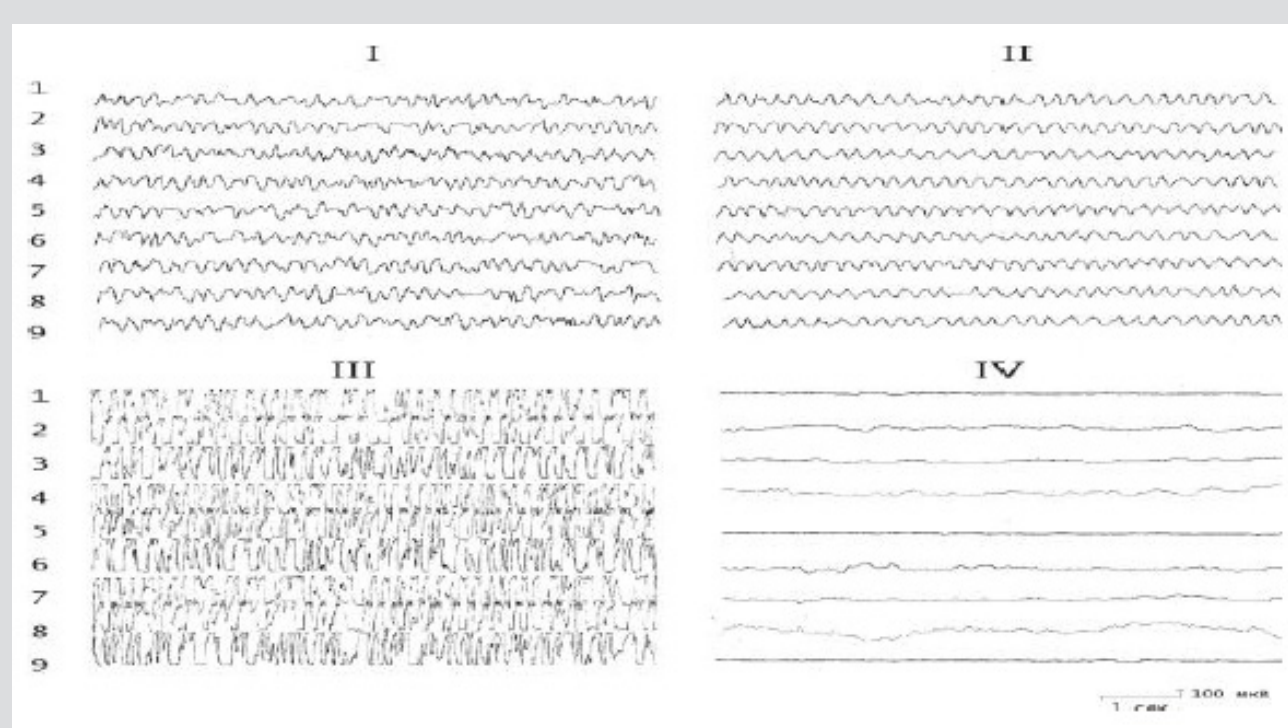

Figure 2: The effect of the introduction of serotonin into the nucleus of the hypothalamus on the electrical activity of the hippocampus before and after the destruction of the DAP. I - background activity; II - after application of 10-30 $\mu$ g of serotonin; III- after application of 50-100 $\mu$ g of serotonin; IV -application of serotonin on the background of the destruction of the DAP. The rest of the notation is the same as in Figure 1. 
The results of these experiments showed that the baseline hippocampal and septal EEG demonstrated irregular activity dominated by oscillations in the range 4-6 Hz. Application of test substances to the dorsal hippocampus before lesioning of the stria terminalis led to ambiguous results. In particular, the effects of biogenic monoamines ultimately led to a redistribution of the peak of the EEG amplitude frequency characteristic in the hippocampus. Thus, while serotonin increased the EEG in the region 5-6 $\mathrm{Hz}$ (Figures 1 \& 2), noradrenaline displaced the peak of the frequency characteristic to the region 4-5 Hz (Figures 2 \& 3). The effects of strychnine and carbachol were rather different. In this experimental situation, carbachol (like strychnine) resulted in generation of highamplitude, regular theta waves of frequency 6-7.5 Hz at different time points, which with time could transform into epidischarges (Figure 3). The EEG changes seen after carbachol and strychnine started in all leads simultaneously and were seen for prolonged periods of time (the maximum observation period was $3 \mathrm{~h}$ ).

\section{I}

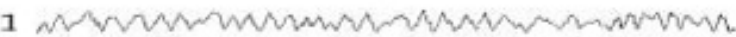

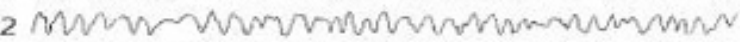

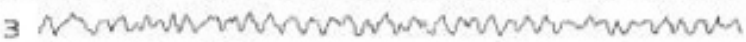

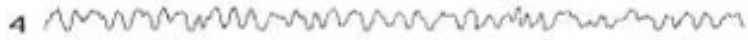

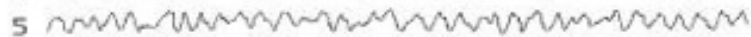

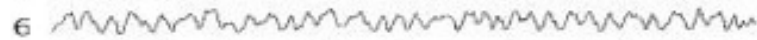

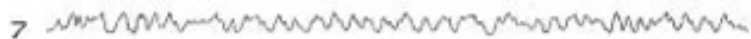

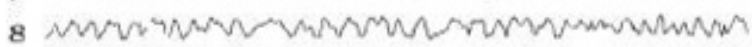

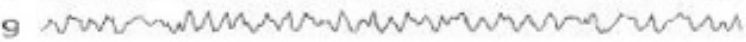

\section{III}

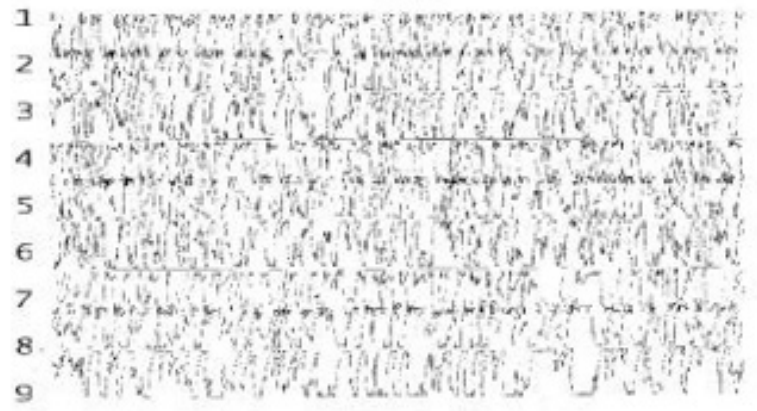

II

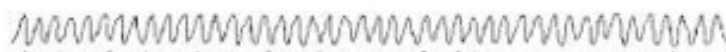

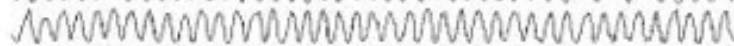

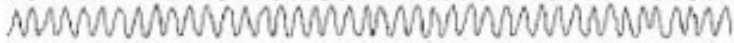

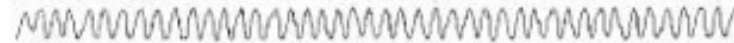

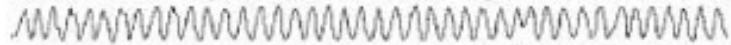

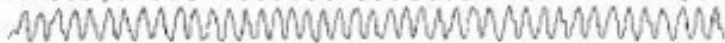

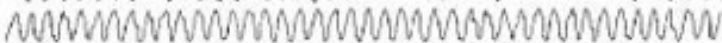

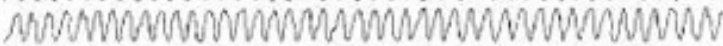

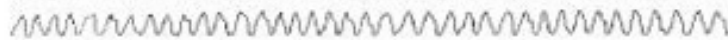

IV

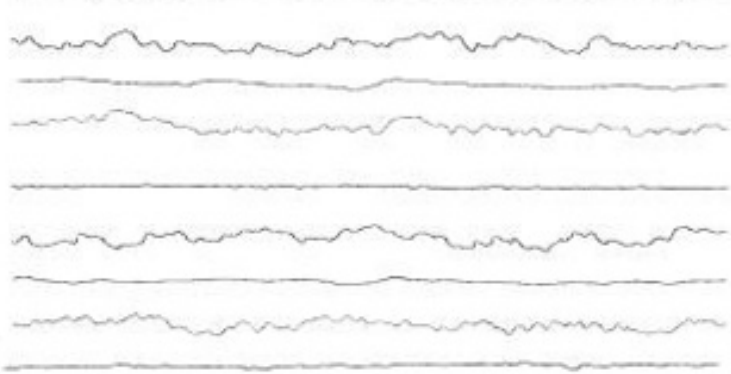

1 cen. 100 unes

Figure 3: The effect of the introduction of carbachol into the nucleus of the hypothalamus on the electrical activity of the hippocampus before and after the destruction of the DAP. I - background activity; II - after application of 0.5-2 $\mu \mathrm{g}$ carbachol; III- after application of $3 \mu \mathrm{g}$ of carbachol; IV -application of carbachol on the background of the destruction of the DAP. The rest of the notation is the same as in Figure 1.

Destruction of dorsal amigdalofugal ways unlike ventral leads to a complete and irreversible blockade of hippocampal theta rhythm (Figure 4). Dynamic observations of the EEG after unilateral lesioning of the DAP showed that the onset of EEG depression often started before the transition period, which showed transient epileptiform activity which subsequently disappeared, leaving only super slow oscillations. Administration of biogenic monoamines into the hippocampus after lesioning of the stria terminalis did not induce any changes at all. Recovery of the electrical activity of the hippocampus due to electrical stimulation of various extrahippocampal structures (mRF, hypothalamus, amygdala) did not occur, while stimulation of the hippocampus itself produced only epidischarges when the maximum stimulation current was used. In this situation, there was a tendency to recovery of the overall activity of the hippocampus and septum, with some features consisting of short-lived (20-30 sec) periodically repeated generation of regular rhythmic activity in the range $0.6-7.5 \mathrm{~Hz}$. Attention is drawn to the fact that, on the one hand, recovery of electrical activity in the hippocampus and septum occurred spontaneously in all leads, while on the other, there was marked synchronicity in the generation of electrical activity. The effects of carbachol and strychnine were largely similar and were longlasting. In addition, analysis of behavioral reactions provided evidence that conditioned reflex responses persisted in conditions of lesioning of the stria terminalis, with only an element of increase in the latent transfer of the response to the conditioned signal (2.02.5sec as compared with 1.0-1.5 sec before lesioning). 


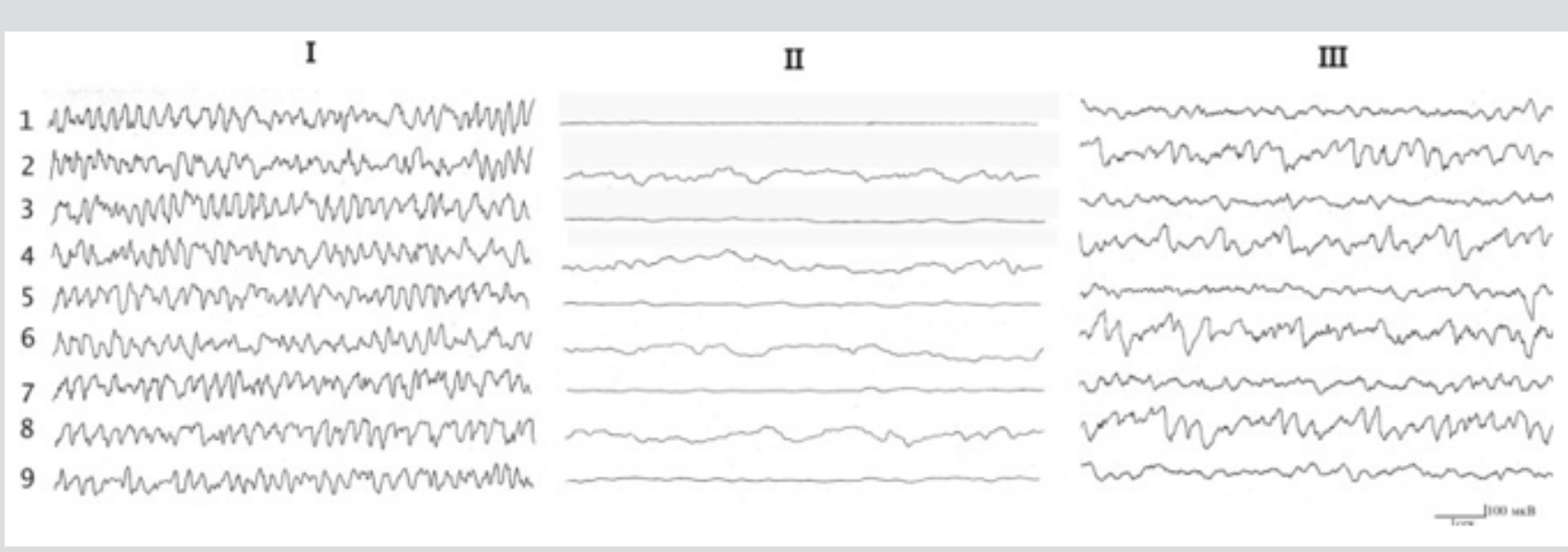

Figure 4: Changes in hippocampal electrical activity in rabbits under conditions of destruction of the dorsal and ventral amigdalofugal ways. Ibackground; II- after destruction of the dorsal amigdalofugal way; III-after destruction of the ventral amigdalofugal way. The rest of the notation is the same as in Figure 1.

Thus, the analysis of our data allows us to conclude that the actions of various neurochemicals in the EEG activity of the hippocampus have a number of common and distinct properties. The former include:

1. The occurrence of synchronized theta wave activity;

2. The absence in different areas of the hippocampus differentiation of bioelectric reactions;

3. Violation of the regularity of the theta-wave activity and the emergence of epidischarges by increasing the dose injected into the structure of the brain monoamines investigated.

For properties that distinguish the action applied neurochemical agents include:

a) The emergence of dominant frequency 6-7.5 number/s cholinergic 5-6 count/s - with serotonergic and 4-5 count/s noradrenergic stimulation of the nucleus the amygdale, hypothalamus, midbrain reticular formation, the medial nucleus of the septum and hippocampus;

b) A change in the amplitude of oscillation synchronized potential compared to background EEG activity, which reached its peak on the background of holinomimetiks, average - with the introduction of 5-HT and was below the background level after administration of NA.

Consider the available data in the literature about the importance of studying brain structures in the regulation of the pituitary-adrenal cortex, it can be assumed that changes in the excitability of hippocampus neurons caused by different electrical and neurochemical effects on the investigated structures of the limbic system, due, apparently, containing different concentrations of endogenous corticosteroids in the blood, and seek to compensate for changes caused by the body. The regulation of the pituitaryadreno-cortical system can participate variety of transmitters of nerve impulses (acetylcholine, NA, 5-HT, dopamine, GABA, prostaglandins, etc.) [10]. According to existing literature data, under the influence of large amounts of corticosteroids in the blood occurs in the hippocampus rhythmic activity with a frequency count of 4-6 count / s, and for topical application of cortisone or hydrocortisone significantly increases the level of the hippocampus pyramidal cells of the hippocampus excitability and formed therein seizure activity [11,12], which, according to the authors, is proof of the direct action of corticosteroids on the dendrites of hippocampus pyramids. In our experiments in hippocampus EEG as synchronized activity recorded frequency 4-6 count/s. However, under the influence of various neurochemicals in the hippocampus recorded respective frequencies: wave number in the range of 4-5 count/s occur when activating noradrenergic systems; 5-6 count/s - the serotonergic system; 6-7.5 number/s - the cholinergic system of the brain. Consider the available data in the literature about the nature and mechanism of action of central neurotropic funds adrenocorticotropic function of the pituitary gland, a comparison with the results of our research led us to the possible involvement of one or another mediator biochemical system in the regulation of different frequencies hippokampal theta rhythm, which reflects different level of activation of the hypothalamic-pituitary system. The results suggest that the regulation of hippocampus-pal theta rhythm, as well as the functional activity of the hypothalamicpituitary system, is polimediatory character and is not determined strictly by a single brain monoaminergic mechanism, ensuring the reliability of the pituitary-adrenal response to these pressures, because the answer this is very important to maintain homeostasis. This, obviously, are huge compensatory abilities of the CNS. A complete and irreversible blockade hippocampal EEG induced destruction DAP clearly indicates that under the given conditions of the hypothalamic-pituitary system is at low level-violation formation rate of secretion of ACTH and corticosteroids. Thus, the results of the research indicate the modulating effect of the limbic 
brain structures in the hippocampal theta rhythm and obviously on the hypothalamic-pituitary system, as well as activating the role of the amygdale in the activity of hypothalamic neurons. All above indicates that a prerequisite for the regulation of excitability of hippocampal neurons is the integrity of the amygdale-hypothalamic connections through which the regulatory effect on the activity of the amygdale hypothalamic neurosecretory cells.

\section{References}

1. Kitchigina VF (2006) Regulation mechanisms and functional significance of theta oscillations in the septo-hippocampal system of the brain. Abstract of dissertation for the degree of Doctor of Biological Sciences, Moscow, Pushchino.

2. Kichigina VF, Kutyreva EV (2002) Modulation of theta activity in the septo-hippocampal system by2-adrenoreceptor agonist clonidine. $\mathrm{Zh}$ Vyssh Nerv Deiat Im I P Pavlova 52: 195-204.

3. Kitchigina V, Popova I, Sinelnikova V, Malkov A, Astasheva E, et al. (2013) Disturbances of septohippocampal theta oscillations in the epileptic brain: Reasons and consequences. Experimental Neurology, 247: 314327.

4. Mysin IE, Kitchigina VF, Kazanovich Y (2015) Modeling synchronous theta activity in the medial septum: key role of local communications between different cell populations. J Comput Neurosci 39: 1-16.

ISSN: 2574-1241

DOI: $10.26717 /$ BJSTR.2019.20.003410

Rafiga Mazahir Baghirova. Biomed J Sci \& Tech Res

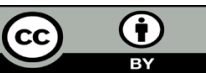

This work is licensed under Creative

Commons Attribution 4.0 License

Submission Link: https://biomedres.us/submit-manuscript.php
5. Steriade M (1996) Arousal: Revisiting the reticular activating system. Sciense 272: 225-226.

6. Smythe JW (1991) Hippocompal theta field activity and theta-on/thetaoff all discharges are controlled by an ascending hypothalamo-septal pathway. J Neuroscience 11: 2241-2248.

7. Berridge CW, Espana RA (2000) Synergistic sedative effects of nonadrenergic alpha1- and beta-receptor blockade in forebrain electroencephalographic and behavior indices. Neuroscience 99: 495505 .

8. Vertes RP (1992) PHA-L analysis of projections from in the supramammillary nucleus in the rat. J Comp Neurology 326: 595-622.

9. Gasanov GG, Kasimov AE, Bagirova RM (1989) Contributions of the amygdala and hypothalamus to the formation of hippocampal theta activity. J Biol Nauki 3: 51-53.

10. Sapronov NS (1998) Pharmacology of the hyperphoresis-adrenal system. Special lit pp. 336.

11. Lishshak K, Endreci E (1967) Neuroendocrine regulation of adaptation activity. Budapest: Academy of Sciences of Hungary pp. 145.

12. Endroczi E (1972) Role of glucococrticoids in controlling pituitaryadrenal function. Acta med Acad Scient Hung 29: 49-59.

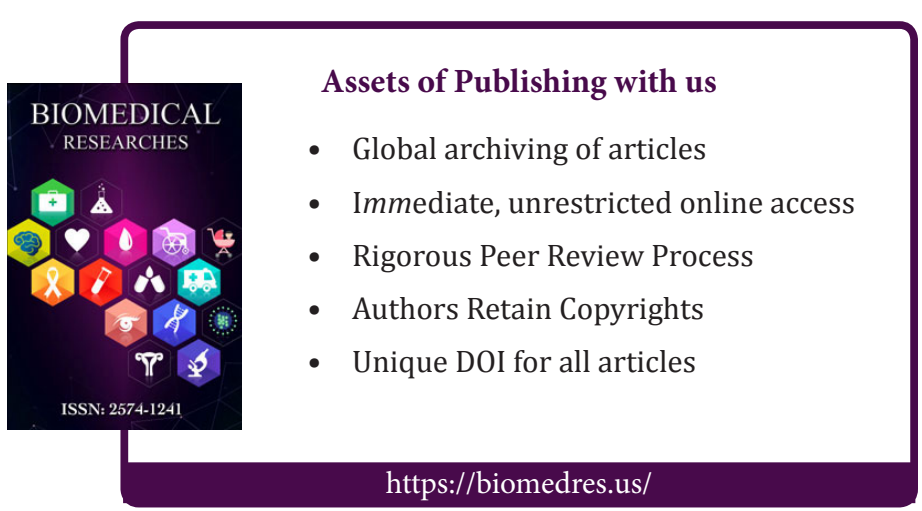

